\title{
Mapeo del servicio ecosistémico de alimento asociado a la pesca en los humedales interiores de Colombia
}

\author{
María H. Olaya Rodríguez ${ }^{1, \bowtie}$; María D. Escobar Lizarazo²; Alexi Cusva33; Carlos A. \\ Lasso Alcalá ${ }^{4} \&$ María C. LONDOÑo Murcia ${ }^{5}$ \\ ${ }^{1}$ Laboratorio de Biogeografía Aplicada, Programa de Evaluación y Monitoreo de la Biodiversidad, Instituto de Investigación de \\ Recursos Biológicos Alexander von Humboldt. Bogotá, Colombia. ${ }^{2}$ Programa de Pós-graduação em Recursos Pesqueiros nos \\ Trópicos, Universidade Federa do Amazonas, Manaus, Brasil. ${ }^{3}$ Grupo de investigación Espacio, Tecnología y participación \\ (ESTEPA), Departamento de Geografía, Facultad de Ciencias Humanas, Universidad Nacional de Colombia. Bogotá, Colombia. \\ ${ }^{4}$ Programa Ciencias Básicas de la Biodiversidad, Instituto de Investigación de Recursos Biológicos Alexander von Humboldt. \\ Bogotá, Colombia. ${ }^{5}$ Programa de Evaluación y Monitoreo de la Biodiversidad, Instituto de Investigación de Recursos Biológicos \\ Alexander von Humboldt. Bogotá, Colombia.
}

\begin{abstract}
Resumen. El recurso pesquero enfrenta grandes amenazas a nivel mundial, por lo que es de vital importancia analizar su dinámica como alimento y su conservación, como parte integral de la evaluación de un servicio ecosistémico (SE). En los humedales interiores de Colombia, la pesca continental es una actividad de importancia cultural y económica pues $\sim 26 \%$ del país está cubierto por humedales. Este trabajo identifica y mapea, a partir de la actividad pesquera continental, los beneficiarios y la dinámica espacial del SE de alimento asociado a la pesca, ofrecido por los humedales interiores de Colombia. Se caracterizan: i) riqueza de las especies asociadas a la pesca, ii) áreas de suministro del SE, iii) áreas de beneficiarios del SE, iv) flujos del SE, y v) estado de amenaza de las especies que componen el recurso pesquero. Este método permite identificar de manera espacialmente explícita las áreas donde se configura el SE, que merecen atención especial en los procesos de gestión del territorio. Se identificaron 138 especies que las comunidades consumen como recurso pesquero. Las cuencas del Orinoco y Amazonas fueron las áreas con mayor riqueza de recursos pesqueros, mientras que las cuencas del Magdalena-Cauca fueron las de mayor suministro del SE. Por su parte, varios municipios de la región andina y amazónica fueron identificados como los principales beneficiarios directos del SE (autoconsumo), en tanto que ciudades principales, seguidas de numerosos municipios vecinos, fueron identificadas como áreas de beneficiarios indirectos (comercialización de la pesca). La espacialización y visualización de estos aspectos permitió analizar los diferentes factores que determinan y afectan el SE en los humedales del país y realizar sugerencias sobre acciones de manejo y gestión del recurso pesquero.
\end{abstract}

[Palabras clave: cuencas, peces de consumo, riqueza, áreas de suministro, áreas de beneficiarios, flujos de pesca, especies amenazadas]

\begin{abstract}
Aвstract. Mapping the ecosystem service of food associated with fishing in inland wetlands of Colombia. The fishery resource faces great threats worldwide, so it is vital to make an analysis of its dynamics as food and its conservation as an integrated part of the evaluation of an ecosystem service (SE). In the inland wetlands of Colombia, fishing is an activity of cultural and economic importance since about $26 \%$ of the national territory is covered by wetlands. This work identifies and maps, from the continental fishing activity, the beneficiaries and the spatial dynamics of the ES of food associated with fishing offered by inland wetlands of Colombia.We describe: i) species richness associated with fishing, ii) areas supplying the ES, iii) areas with the beneficiaries of the ES, iv) flow of the ES, and v) the endangered status of the species within the fishery resource. We identified 138 fish species were consumed by communities. The Orinoco and Amazon basins were identified as the richest areas of fishery resources species, whereas the Magdalena-Cauca's basins were identified as the areas with more supply of the ES. On the other hand, several communities in the Andean and Amazonian region were identified as the main direct beneficiaries of ES (self-consumption), while main cities, followed by numerous neighboring municipalities, were identified as areas of indirect beneficiaries (marketing of fishery). Spatialization and visualization of these aspects allowed to analyze the different factors that determine and affect the ES in the country's wetlands and make suggestions on management actions for the fishery resource.
\end{abstract}

[Keywords: watersheds, fishes for consumption, richness, supply areas, areas of beneficiaries, flows of fishing, endangered species]

Editor asociado: Lucas Garibaldi

$\triangle$ molaya@humboldt.org.co
Recibido: 10 de abril de 2016

Aceptado: 29 de octubre de 2016 


\section{INTRODUCCIÓN}

De los servicios de provisión que ofrecen los humedales, el pescado y sus productos derivados son servicios importantes de las aguas continentales (MEA 2005). En la mayoría de las zonas rurales de países en vía de desarrollo, la pesca continental desempeña una función clave como sustento de los medios de vida al contribuir con la generación de empleo, la seguridad alimentaria y mitigación de la pobreza (FAO 2016). La contribución de la pesca continental registrada para 2014 mostró que se produjeron $\sim 12000000 \mathrm{t}$ de pescado, que corresponden a $\sim 13 \%$ de la pesca mundial durante ese año (FAO 2016). A pesar de estas cifras, se cree que la cantidad de pesca continental real es superior a la cifra oficial ya que la mayor parte de los registros son incompletos debido a la dificultad para el registro de información (Barletta et al. 2016; FAO 2016).

En Colombia, el 26\% del territorio continental está cubierto por humedales (Flórez-Ayala et al. 2015), que se reconocen por su comportamiento dinámico entre las sequías y las inundaciones.Esta característica le ha conferido al país el mote de "Colombia anfibia" (Jaramillo 2015). Se ha identificado que la actividad pesquera para el consumo dentro de estos sistemas de humedales contribuye con el $16.6 \%$ del total de la actividad pesquera nacional, que incluye la pesca marítima y la actividad de acuicultura (CCI e INCODER 2011). A su vez, este porcentaje de pesca benefició 75000 familias que viven en los sistemas de humedales (Arias 2013; Gutiérrez et al. 2011a). Por lo general, dichos servicios son muy vulnerables a los cambios ambientales y a conflictos socioeconómicos, tales como los efluentes dela agricultura, ganadería y minería, sobrepesca, alteración y transformación del hábitat, introducción de especies exóticas, construcción de infraestructura hídrica (embalses, hidroeléctricas, canales, etc.) y cambio climático (Gutiérrez et al. 2011b; Valderrama et al. 2011). Por lo tanto, estos factores amenazan la seguridad alimentaria de las poblaciones ribereñas y de las regiones e incrementan el deterioro de los ecosistemas y la pérdida de la biodiversidad (Arias 2013).

Frente a estos escenarios de amenazas al ecosistema de humedales interiores, el gobierno colombiano reconoció la necesidad de conocer las dinámicas que existen entre los humedales y sus pobladores para enfocar las acciones de gestión y conservación (Jaramillo et al. 2015). La ubicación espacialmente explícita de los beneficios que los ecosistemas ofrecen a la sociedad brinda un insumo fundamental para la toma de decisiones (Martínez-Harms and Balvanera 2012). Por medio de este método se identifican las áreas en donde se concentran los servicios ecosistémicos y, en consecuencia, se caracterizan aquellas zonas que merecen una atención especial para la conservación y / o manejo integral del ecosistema y el recurso (Balvanera 2001; Daily et al. 2009; MartínLópez 2012). Además, el método permite monitorear la implementación de políticas de gestión a través de escalas espaciales y temporales, de manera que se pueda estimar el éxito del manejo y la conservación de los servicios ecosistémicos (Crossman et al. 2013; García-Nieto et al. 2013; Maynard et al. 2010; Mubareka et al. 2013).

En Latinoamérica, el uso de información espacialmente explicita para mapear servicios ecosistémicos es un campo de estudio aún en desarrollo (Crossman et al. 2013; Egoh et al. 2012; Martínez-Harms and Balvanera 2012). En este sentido, y bajo el contexto de la caracterización de los humedales interiores de Colombia, se desarrolló el mapeo del servicio ecosistémico de alimento asociado a la pesca continental para así identificar de forma espacialmente explícita la riqueza y el estado de conservación del recurso pesquero, las áreas geográficas que proveen y se benefician del SE, y determinar su flujo desde su obtención hasta su consumo. Esto nos permitió ver algunos factores intrínsecos y extrínsecos que intervienen en la configuración del SE, y asimismo apoyar varias recomendaciones sobre la gestión y el manejo del recurso pesquero.

\section{MAteriales y Métodos}

\section{Área de estudio}

El área de estudio fue la parte continental de Colombia, que corresponde a 1100000 $\mathrm{km}^{2}$ (-79.011 O; 12.46 N; -66.85 E; -4.23 S).De toda esta superficie, el $26 \%$ corresponde a humedales interiores (Flórez-Ayala et al. 2015). El territorio nacional se divide en cinco grandes macrocuencas: Caribe $\left(102786 \mathrm{~km}^{2}\right)$, Magdalena-Cauca $\left(271118 \mathrm{~km}^{2}\right)$, Orinoco $\left(347208 \mathrm{~km}^{2}\right)$, Pacífico $\left(77302 \mathrm{~km}^{2}\right)$ y Amazonas (341991 km²), que a su vez contienen 31 cuencas hidrográficas. Además, el país está dividido políticamente en 32 departamentos que contienen 1149 municipios. 


\section{Riqueza de especies}

Para describir la riqueza de especies de peces, se utilizaron capas vectoriales previas de las áreas de distribución de las especies identificadas como recursos pesqueros en Colombia (Lasso et al. 2011), las cuales fueron sobrepuestas e intersectadas por medio de un proceso de análisis espacial en un sistema de información geográfica (SIG) con las capas de las cuencas hidrográficas del país. A partir de este proceso se estimó la riqueza de especies (número de especies) en cada una de las cuencas hidrográficas.

Para describir las áreas de fuente y destino del SEse identificaron dos tipos de áreas en el territorio colombiano: de suministro y de beneficiarios (Syrbe and Walz 2012).

\section{Áreas de suministro del servicio ecosistémico (ASSE)}

Las ASSE representan los lugares donde se genera el servicio ecosistémico. Sus límites son principalmente naturales o biofísicos (e.g., unidades ecológicas o cuencas hidrográficas) (Syrbe and Walz 2012). Para trazar los límites de estas áreas se utilizaron dos fuentes de información: el mapa de cuencas hidrográficas de Colombia y los datos suministrados por las estadísticas pesqueras nacionales (CCI e INCODER 2011) sobre toneladas de pesca capturadas durante el 2011. Estos últimos son colectados en diversos centros de acopio contenidos dentro de las cuencas hidrográficas. En el caso particular de la macrocuenca del Amazonas, se contó además con información de toneladas de pesca de subsistencia para el año 2011 (Agudelo et al. 2011).

\section{Áreas de los beneficiarios del servicio ecosistémico (ABSE)}

Representan los lugares donde se demanda el SE y son complementarias a las ASSE (Syrbe and Walz 2012). A diferencia de los anteriores, sus límites están relacionados con las dinámicas sociales y por lo tanto se asocian mejor con unidades administrativas. Las ABSE pueden clasificarse en directas o indirectas. Las directas están ubicadas dentro de las ASSE (i.e., donde los pescadores tienen una fuerte relación cultural con la actividad pesquera y consumen los mismos recursos pescados) (Figura 1). Las indirectas se encuentran separadas espacialmente de las áreas de suministro, y el flujo está determinado por la

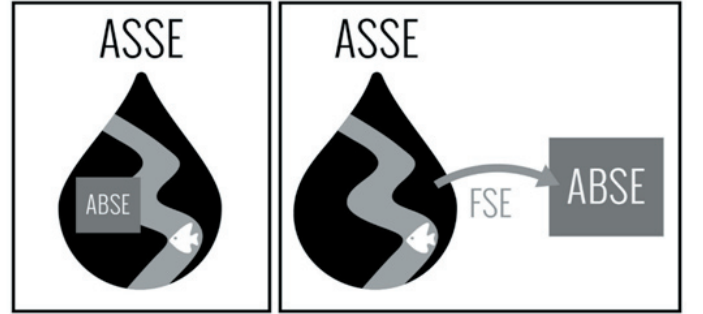

Figura 1. Definiciones de las áreas de beneficiarios directos de los servicios ecosistémicos directos (izquierda) y las áreas de beneficiarios indirectosde los servicios ecosistémicos indirectos (derecha). ASSE=áreas de suministro del servicio ecosistémico; ABSE=áreas de los beneficiarios del servicio ecosistémico; FSE=flujo del servicio ecosistémico.

Figure 1. Definitions of areas with direct beneficiaries of the ecosystem services (left) and areas with indirect beneficiaries of the ecosystem services (right). ASES = Areas supplying the ecosystem service; ABSE = Areas of the beneficiaries of the ecosystem service; FES = Flow of ecosystem service.

comercialización del recurso (Figura 1). Para identificar los límites de estas áreas se utilizó el mapa de municipios de Colombia y las estadísticas pesqueras (CCI e INCODER 2011) sobre toneladas de pesca procesadas para su comercialización y consumidas localmente por municipio en el año 2011.

\section{Flujo del servicio ecosistémico (FSE)}

Se define como las conexiones entre las áreas de suministro con las de beneficiarios. Se cuantificó a través de las toneladas de pesca comercializadas desde las áreas de suministro hacia las de beneficiarios, tanto directos como indirectos. El flujo fue construido con la información de toneladas de pesca (CCI e INCODER 2011; Agudelo et al. 2011) capturadas y demandadas a nivel de cuencas hidrográficas y de ciudades capitales y municipios para el año 2011. Los flujos se esquematizaron mediante flechas que indican la dirección y las toneladas de pescado comercializadas. Para ello, se utilizó el paquete Circlize (Gu 2014) bajo el lenguaje de programación R ( $R$ Development Core Team 2011).

\section{Estado de conservación de las especies consideradas recurso pesquero}

Se tomó como base las categorías de amenaza definidas en el libro rojo de peces dulceacuícolas de Colombia (Mojica et al. 2012) y las toneladas de captura de cada una de esas especies a partir de las estadísticas pesqueras (CCI e INCODER 2011). Con estas 
dos fuentes de información se generó un mapa a nivel de las grandes macrocuencas de Colombia donde se cuantificó la importancia relativa de las especies amenazadas respecto al total de la actividad pesquera. Todos los mapas fueron preparados y editados en el programa ArcGIS 10.2 (ESRI 2013).

\section{RESULTADOS}

\section{Riqueza de especies}

En el conjunto de datos analizados se describieron 138 especies de peces consideradas como recurso pesquero. La mayor riqueza se observó en las zonas hidrográficas de las macrocuencas del Amazonas y el Orinoco, donde se registró la presencia de entre 30 y 68 especies para el consumo alimentario. Por su parte, las cuencas del Caribe, Pacífico y Magdalena-Cauca mostraron los menores valores de riqueza (9 a 29 especies) (Figura $2 \mathrm{~A}$ ).

\section{Áreas de suministro del servicio ecosistémico (ASSE)}

Las cuencas del bajo Magdalena-Cauca-San Jorge y las del bajo y medio Magdalena son

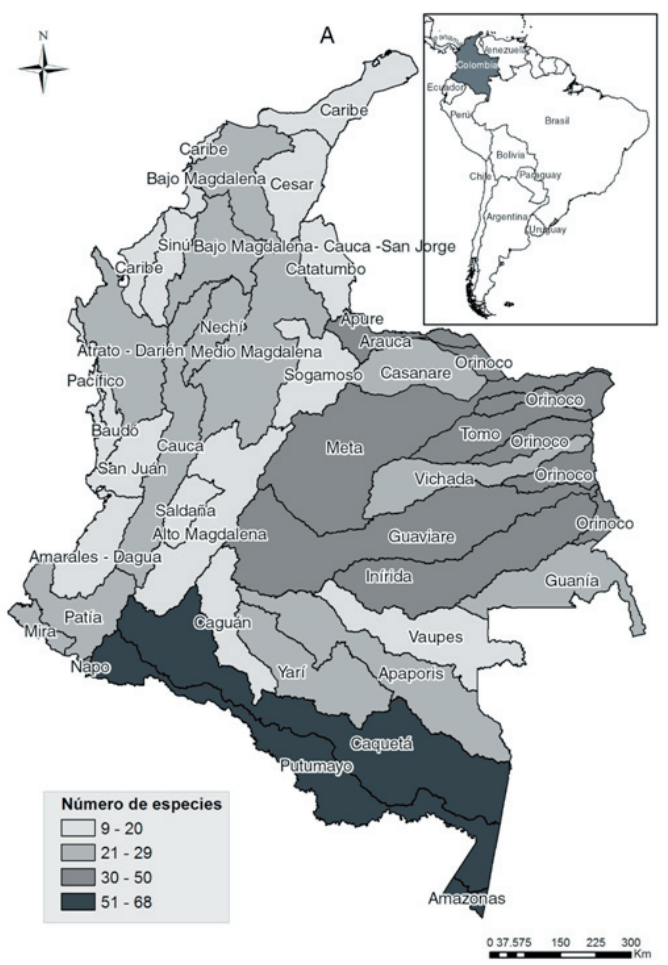

las que brindaron el mayor suministro del SE, junto a la cuenca del Amazonas (entre 3320 y 8847 t). La cuenca del río Atrato-Darién, en la región Pacífica, junto a las demás cuencas de la macrocuenca del Amazonas reportaron entre 387 a 1560 t. Por su parte, las cuencas de la macrocuenca del Orinoco (cuencas del Meta, Inírida, Vaupés y Guaviare) reportaron entre 20 a $386 t$, al igual que la cuenca del Sinú. La cuenca con menor suministro fue la del Vichada con un valor entre 1 a 9 t (Figura 2B).

\section{Áreas de los beneficiarios del servicio ecosistémico (ABSE)}

ABSE directos. En el análisis se destacan los municipios de Barrancabermeja en el medio Magdalena, Magangué en el bajo Magdalena, Quibdó en la cuenca del Atrato, Puerto Leguízamo, Solano y Puerto Nariño en la cuenca del Amazonas, donde la productividad pesquera fue entre 371.6 a $1127 \mathrm{t}$. El servicio también favorece a otras comunidades como El Plato, Caucasia, El Banco en el bajo Magdalena, Honda y Puerto Boyacá en el medio Magdalena, Lorica en el bajo Sinú, Turbo en el bajo Atrato, Arauca e Inírida en el



Figura 2. Mapas de (A) riqueza de especies asociadas al recurso pesquero y (B) las áreas de suministro del servicio ecosistémico (ASSE) de provisión de alimento asociado a la pesca en las cuencas hidrográficas de Colombia.

Figure 2. Maps of (A) species richness associated with the fishery resource and (B) areas supplying the ecosystem service (ASES) of food associated with fishing in the watersheds of Colombia. 

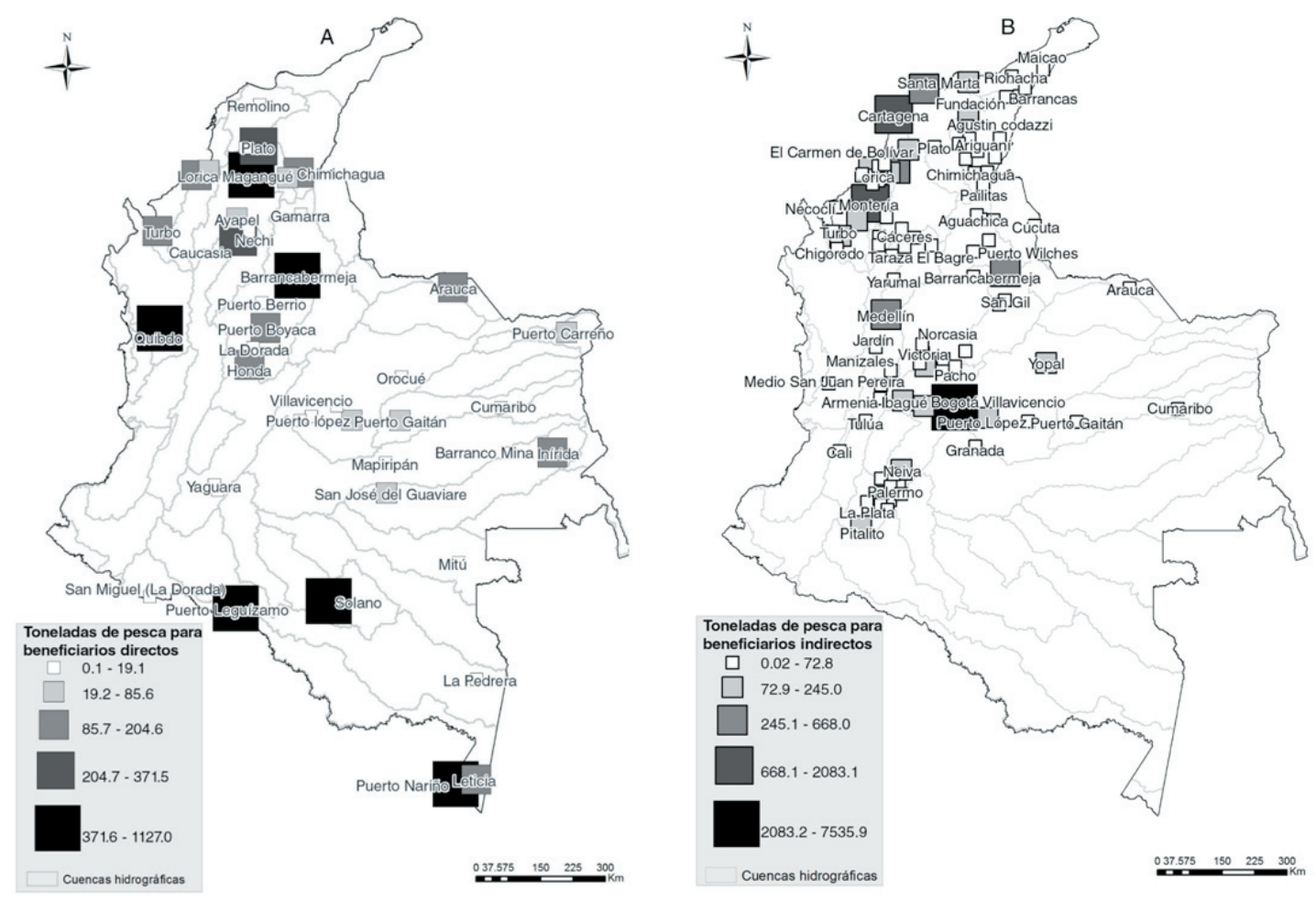

Figura 3. Mapas de (A) las áreas de beneficiarios directos y (B) beneficiarios indirectos del servicio ecosistémico de provisión de alimento asociado a la pesca en las cuencas hidrográficas de Colombia.

Figure 3. Maps of (A) areas of direct beneficiaries and (B) indirect beneficiaries of the ecosystem service of food associated with fishing in the watersheds of Colombia.

Orinoco y Leticia en el Amazonas entre otros, donde el recurso pesquero también representa una proporción importante de la dieta de sus pobladores (Figura 3A).

ABSE indirectos. Las ciudades capitales fueron las más beneficiadas por el servicio de los humedales. A Bogotá llegaron entre 2083 y 7540 t de pescado (29\% capturado en los sistemas dulceacuícolas del país), seguida por Medellín y Bucaramanga. Las ciudades caribeñas también revelaron un consumo elevado de pescado, entre las que se destacan Montería y Cartagena, con un consumo entre 668 y 2083 t, y Barranquilla y Sincelejo, con valores entre 245 y $668 \mathrm{t}$. Poblaciones de menor tamaño, principalmente en la región Andina, mostraron su gran dependencia por el recurso pesquero (Figura 3B).

\section{Flujo del servicio}

El flujo de pesca mostró cómo en el 2011 la cuenca del Magdalena abasteció a gran parte de poblados de su propia región y otras cuencas como el Sinú y el Caribe (Figura 4A). Por otra parte, mostró como Bogotá fue abastecida por Leticia, así como por ciudades ubicadas en el medio y bajo Magdalena (Figura 4B). Por su parte, las ciudades caribeñas como Barranquilla, Cartagena y Montería fueron abastecidas por los municipios ubicados en el departamento de Bolívar (Figura 4B).

\section{Estado de conservación}

De las 138 especies de peces para el consumo registradas en las capturas para el año 2011,27 especies fueron identificadas con algún grado de amenaza (crítica, en peligro, vulnerable y casi amenazada). La oferta pesquera para el consumo de la cuenca del Atrato-Darién y la macrocuenca Magdalena-Cauca fue soportada en $90 \%$ y $84 \%$, respectivamente por las especies con algún grado de amenaza. Por su parte, las macrocuencas del Amazonas, Orinoco y la cuenca del Sinú mostraron que su pesquería está sustentada en $46 \%, 49 \%$ y $21 \%$, respectivamente, por especies en algún estado de amenaza (Figura 5).

\section{DISCUSIÓN}

Este ejercicio de mapeo permitió hacer un primer reconocimiento geográfico de 

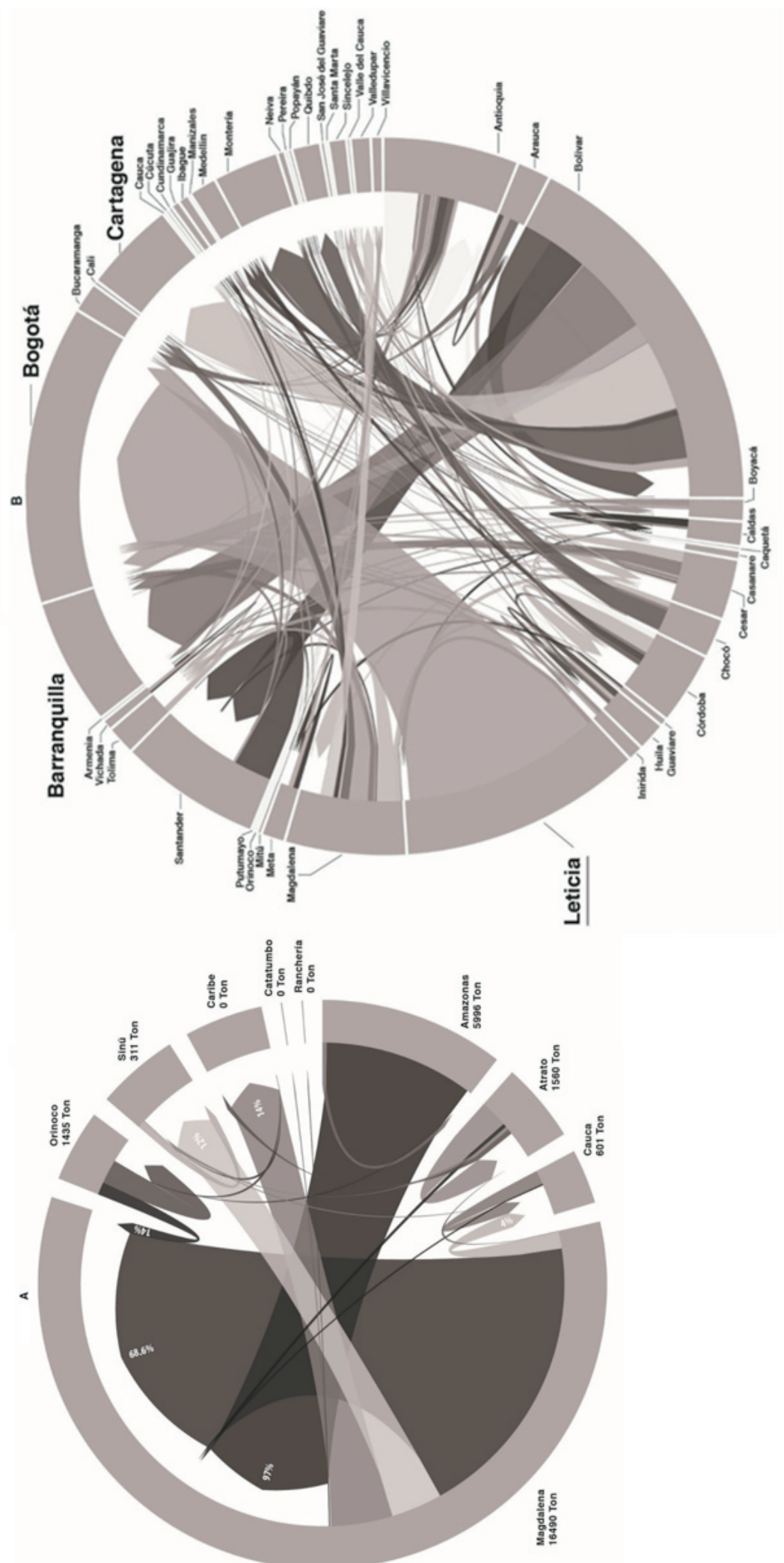

Figura 4. Flujos del servicio ecosistémico (FSE) de provisión de alimento asociado a la pesca por (A) cuencas hidrográficas y (B) ciudades principales y municipios de Colombia.

Figure 4. Flows of ecosystem service (FES) of food associated with fishing in (A) watersheds and (B) major cities and municipalities of Colombia. 


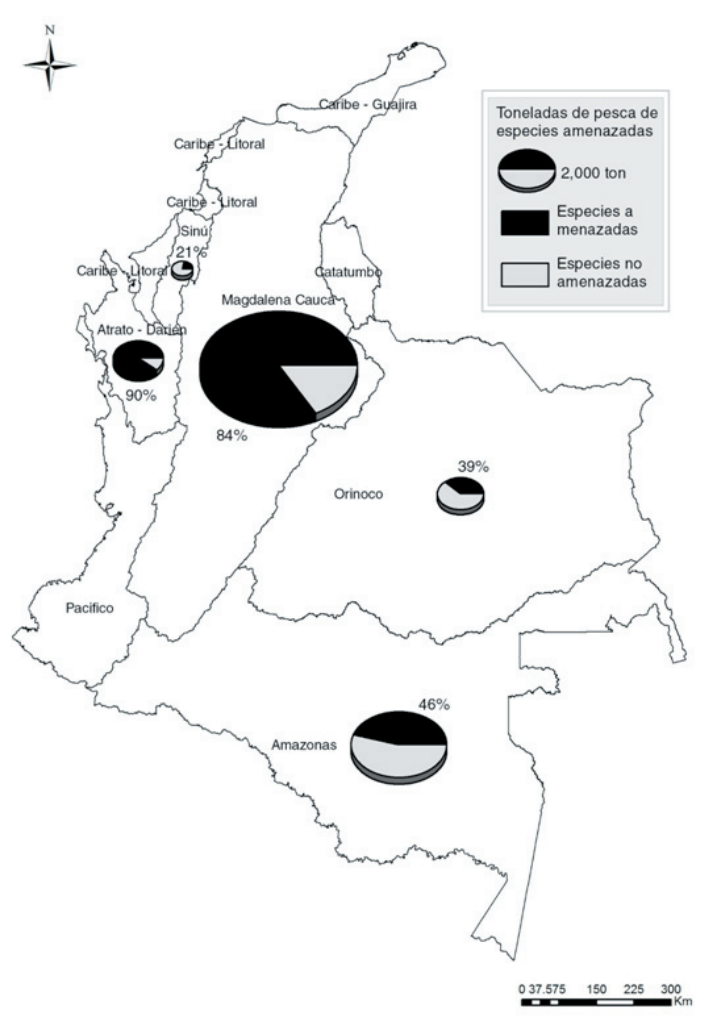

Figura 5. Mapa del aporte (porcentaje) de las especies amenazadas a las pesquerías de las macrocuencas de Colombia.

Figure 5. Map of the contribution (percentage) that threatened species make to fisheries of the macrowatershed of Colombia.

la distribución de la riqueza del recurso pesquero en las zonas hidrográficas de Colombia y de la complejidad del servicio ecosistémico de alimento asociado a la pesca, algo que se relaciona con el hecho de que los servicios ecosistémicos no se presentan de forma homogénea en el paisaje y son dinámicos en el tiempo (Fisher et al. 2009). Dicha complejidad puede ser entendida por una serie de i) factores intrínsecos, que se caracterizan por no ser modificables en el corto plazo y por estar relacionados con el ambiente del recurso pesquero (e.g., las condiciones hidro-geomorfológicas de las cuencas y la configuración espacial de la riqueza de especies [variedad de hábitats y nichos ecológicos y características biológicas y comportamentales de las especies ícticas]), y ii) factores extrínsecos, que se caracterizan por ser modificables en el corto plazo y por estar más relacionados con la actividad humana (i.e., pescadores, centros de acopio, características del consumo de pescado en el país y actividades antrópicas que amenazan la calidad y cantidad del recurso pesquero).

\section{Factores intrínsecos}

Factoreshidro-geomorfológicos.Puedenestar explicando que pese a la menor diversidad de especies, la cuenca del bajo Magdalena-CaucaSan Jorge fue la que suministró mayormente el servicio de provisión de alimento en 2011 (Figura 2A) dado que allí se encuentra el área de convergencia de la parte baja de los ríos Magdalena, Cauca y San Jorge, conocida como la depresión Momposina. Esta zona se caracteriza por la conexión de cadenas irregulares de ciénagas de poca profundidad con el cauce principal a través de pequeños caños, convirtiéndola en un área inundable propicia para la abundante producción de plancton y plantas acuáticas que generan un ambiente apto para la reproducción, cría y protección de los peces y otros organismos acuáticos. La depresión Momposina es la región más baja de la cuenca, donde se concentra $\sim 80 \%$ de las ciénagas del área. Es la principal zona inundable de la macrocuenca Magdalena-Cauca (Galvis and Mojica 2007).

Riqueza de especies. La mayor riqueza de especies en las macrocuencas del Amazonas y Orinoco (Figura 2A), principalmente en las áreas relacionadas a grandes afluentes como los ríos Amazonas, Putumayo, Caquetá, Orinoco, Guaviare, Meta y Arauca, se asocia a que estas dos macrocuencas siempre han sido reconocidas como el núcleo de la riqueza de especies de peces a nivel mundial, y en particular del Neotrópico (James et al. 2011). Por esta razón ofrecen la mayor variedad de especies susceptibles al consumo (Lasso et al. 2011). No obstante, dentro de las mismas macrocuencas existieron variaciones en la riqueza (cuencas de Yarí, Apaporis, Vaupés, Guainía, Vichada y Casanare), relacionadas con el tipo de agua de cada río y sus respectivos afluentes (aguas claras, negras y blancas), así como con la productividad primaria acumulada durante la trayectoria de sus aguas hasta la desembocadura (NúñezAvellaneda et al. 2006). Por el contrario, las cuencas hidrográficas del Magdalena-Cauca, Caribe y Pacífico reflejaron menor riqueza de peces de importancia para el consumo. Este patrón de riqueza coincide con el descrito por Lasso et al. (2011) y se considera una manifestación del tipo de aguas (claras y blancas), variación de latitud y menor área de la cuenca, comparado con los ríos Orinoco y Amazonas. En particular, la macrocuenca Magdalena-Cauca presenta ríos pequeños y torrenciales de alta productividad, lo que provoca que la diversidad de especies de peces 
asociada sea baja y especializada (Galvis and Mojica 2007).

\section{Factores extrínsecos}

Pescadores, centros de acopio y consumo. Las cuencas de mayor suministro (macrocuenca Magdalena-Cauca) es donde residen el mayor número de pescadores artesanales (aproximadamente 43730) (Gutiérrez et al. 2011a) que se benefician de este SE. En este sentido, es posible establecer que las cuencas de la región andina del país se reconocen como las ASSE más representativas, principalmente debido a sus características hidro-geomorfológicas y a la actividad de un gran número de pescadores artesanales que habitan cerca a los humedales de esa región. Estás áreas también se identifican como las ABSE directas más representativas, ya que del total del suministro del servicio proveniente de éstas cuencas, el $68.6 \%$ se queda en la misma región para abastecer a sus pobladores concentrándose principalmente en los municipios de Barrancabermeja, Magangué, Plato y Caucasia (Figura 3A). La segunda ASSE más importante fue la del río Amazonas, que presenta como centro de acopio la ciudad de Leticia, que recibe la pesca proveniente del territorio nacional y la proveniente desde Iquitos (Perú) y de Tefé (Brasil) (Agudelo et al. 2011). Los municipios de Puerto Leguízamo, Solano y Puerto Nariño se destacaron por su alto consumo de pescado, que es un reflejo directo de la importancia del recurso en todas las localidades de la Amazonía colombiana (Figura 3A).

Se observó un gran vacío de información sobre suministro y demanda del SE en varias cuencas hidrográficas de Colombia. Esto puede estar relacionado con que el levantamiento de información de la Autoridad Nacional de Pesca y Acuicultura (AUNAP) fue orientado hacia el monitoreo de los principales puertos pesqueros del país. Por lo tanto, esta información representa un sesgo en la caracterización del SE, con algunos vacíos en regiones donde el recurso pesquero es parte importante de la dieta de los indígenas, ribereños y afrodescendientes, pero que por la deficiencia de la información no pudieron ser representadas geográficamente. Para el caso de las cuencas de la región de la Orinoquía se conoce que una gran cantidad de la población en los municipios y los resguardos indígenas consumen en gran medida el recurso pesquero. Sin embargo, muchos de estos datos no están incluidos en las estadísticas pesqueras debido a que la extensión de la región y la dispersión de su población hace difícil su registro (Ramírez-Gil y Ajiaco-Martínez 2011). El excedente de la pesca de subsistencia por parte de los beneficiarios directos del SE es vendido y distribuido hacia las ABSEs indirectos, conformadas por cuencas y municipios vecinos (Figura 3B), así como por ciudades capitales con fuertes costumbres de consumo de pescado, tales como las ciudades caribeñas (Figura 4A y 4B). La ciudad de Bogotá, que alberga pobladores de todas las regiones del país, es fuertemente abastecida por la región del Amazonas y en menor cantidad de la macrocuenca del Magdalena-Cauca. Aunque se observa que el flujo del servicio pesquero abastece de alimento a la gran mayoría del territorio nacional, cabe mencionar que se desconoce toda su complejidad. Si bien se conoce el primer beneficiario (municipio), el recurso puede tomar otras rutas de comercialización que no son reflejadas por los datos empleados en este estudio.

Otras actividades antrópicas. Cuencas como las del Atrato, Sinú y el Orinoco, identificadas como ASSE de menor magnitud, también pueden estar siendo afectadas por la pérdida de cobertura boscosa, la sustitución de la actividad pesquera por otras actividades económicas (e.g., la explotación petrolera y cultivos ilícitos), el orden público de la región y el poco acceso a los servicios públicos que faciliten la conservación y transporte del recurso pesquero (Ramírez-Gil y AjiacoMartínez 2011). Otros factores que afectan y amenazan la oferta en cantidad y calidad del SE son los excesos de efluentes procedentes de la agricultura, la ganadería y la minería (que afectan el crecimiento y la mortalidad de las especies acuáticas, produciendo acumulación de toxinas en el pescado o bioacumulando metales pesados, insecticidas y fungicidas), la alteración y transformación del hábitat (que incluyen la degradación de la calidad, modificación hidrológica y transformación de las planicies de inundación), la introducción de especies exóticas, el represamiento de ríos para la instauración de infraestructura (embalses, hidroeléctricas, canales, etc.), el cambio climático (para el que se prevé modificaciones en la distribución y la composición de los recursos de la pesca continental dada la modificación en las variables ambientales) y la sobrepesca de los principales recursos pesqueros (FAO 2010; Gutiérrez et al. 2011b).

Varios de estos factores son considerados para la determinación del estado de amenaza de las especies (Mojica et al. 2012), que en el 
caso de la macrocuenca Magdalena-Cauca conforman más del $80 \%$ de su producción pesquera. En este sentido, el mantenimiento del SE contempla dos aspectos fundamentales. Por un lado, tomar medidas de gestión y conservación en los territorios del país para disminuir el impacto negativo de estos factores extrínsecos sobre el recurso pesquero, y, por tanto, sobre la oferta del SE. Gutiérrez et al. (2011b) señalan que para lograr una pesca sostenible es necesario proteger los hábitats clave, en especial para aquellas especies que presentan necesidades ecológicas estrictas (lugares de desove y cría), para así mantener o restaurar las funciones y los procesos ecosistémicos que dan origen a los servicios. Por otra parte, es necesario reconocer que como servicio ecosistémico, las condiciones socioeconómicas de quienes demandan el servicio también pueden estar actuando como una amenaza para su suministro. Gutiérrez et al. (2011c) mencionan que la crisis global que la FAO ha venido anunciando desde hace más de diez años para las principales pesquerías se ha relacionado de manera directa con el aumento de las actividades de la pesca industrial y comercial en el mundo.

Estas actividades han seguido un modelo que acentúa la privatización de los recursos y han llevado a la disminución de los derechos de acceso a los recursos pesqueros, la expulsión de comunidades de pescadores artesanales, el desplazamiento de éstas del campo a la ciudad y la substitución de la actividad pesquera por otras actividades económicas como la minería, la agricultura (monocultivos) y el turismo. Frente a esto, es importante resaltar la necesidad inmediata de reconocer y visibilizar la pesca artesanal como un sector productivo ya que aporta una gran cantidad de proteínas de consumo al país (Figuras $4 \mathrm{~A}$ y 4B). Así mismo, resulta clave impulsar acciones de co-manejo entre el Estado y las organizaciones de pesca artesanal coherentes con el recurso pesquero y con el bienestar de estos productores primarios, para quienes, al contrario de lo que se pensaría, las condiciones de ingresos y nutrición son precarias y de alta vulnerabilidad (Gutiérrez et al. 2011c).

Este ejercicio de mapeo del servicio ecosistémico de alimento asociado a la pesca a escala nacional es el primero que se hace en el país y genera algunas recomendaciones. En primer lugar, es necesario que los registros de las estadísticas pesqueras sean completados para todas las cuencas hidrográficas del país, tanto en términos de suministro del servicio ecosistémico como de su demanda en las poblaciones locales de los humedales interiores. Esto permitirá completar la configuración y la dinámica del SE en todo el territorio nacional. En segundo lugar, es imperante apoyar las acciones locales de conservación y gestión, como es el caso de la priorización de la macrocuenca MagdalenaCauca, donde los datos pusieron en evidencia el suministro elevado y la alta demanda del servicio ecosistémico, cuyo estado puede estar altamente amenazado por el delicado estado de conservación en el que se encuentran las especies que constituyen la actividad pesquera de esta región. Estas acciones deben estar dirigidas a una gestión integral del recurso que contemple un ordenamiento territorial acorde a las necesidades de las comunidades locales y que contribuya a establecer un nuevo modelo pesquero que combata la sobreexplotación que ha impulsado el modelo actual (Gutiérrez et al. 2011b).

Por último, este tipo de trabajos son fundamentales para plantear la línea base de monitoreo para este servicio ecosistémico, que debe ser complementada con estudios posteriores de evaluación y caracterización de funciones y procesos ecosistémicos y estudios de valoración. Esto permitirá elaborar herramientas para mejores prácticas en la gestión del SE y la conservación del recurso que lo genera.

Agradecimientos. Deseamos expresar nuestros sinceros agradecimientos a los expertos E. Agudelo-Córdoba, G. PoloRomero, J. C. Alonso, L. F. Jiménez, M. Valderrama-Barco, P. Sánchez y R. E. AjiacoMartínez por sus valiosas sugerencias para el mejoramiento de este trabajo. A los miembros del Laboratorio de Biogeografía Aplicada J. Velásquez, C. Gutiérrez, I. González y D. López por el apoyo y aportes en el desarrollo de este trabajo. A J. Agudelo y L. Mesa por su contribución con los mapas de distribución de especies de peces. A la Autoridad nacional de acuicultura y pesca (AUNAP) por proveer los datos con los cuales se realizó este estudio. A J. C. Bello, O. Orrego y L. Nägele por apoyar esta investigación en sus inicios. Este proyecto fue financiado por elConvenio Interadministrativo No.005 de 2013 entre el Fondo Adaptación y el Instituto de Investigación de Recursos Biológicos Alexander von Humboldt. 


\section{REFERENCIAS}

Agudelo, E., C. L. Sánchez, C. F. Rodríguez, C. A. Bonilla-Castillo, and G. A. Gómez. 2011. Diagnóstico de la pesquería en la cuenca del Amazonas. Capítulo 5. Pp. 143-166 en: C. A. Lasso, F. P. Gutiérrez, M. A. Morales-Betancourt, E. Agudelo, H. Ramírez-Gil, et al. (eds.). Pesquerías continentales de Colombia: cuencas del Magdalena-Cauca, Sinú, Canalete, Atrato, Orinoco, Amazonas y vertiente del Pacífico. Serie Editorial Recursos Hidrobiológicos y Pesqueros Continentales de Colombia. Instituto de Investigación de Recursos Biológicos Alexander von Humboldt, Bogotá D. C., Colombia.

Arias, P. A. 2013. Bases de política para el sector pesquero y la acuicultura en Colombia. Informe Técnico. Dirección de Pesca y Acuicultura-DPA, Ministerio de Agricultura y Desarrollo Rural-MADR y Corporación Colombia Internacional -CCI. Bogotá D.C., Colombia. Pp. 178.

Balvanera, P. 2001. Conserving biodiversity and ecosystem services. Science 291(5511):2047-2047.

Barletta, M., V. E. Cussac, A. A. Agostinho, C. Baigún, E. K. Okada, et al. 2013. Fisheries ecology in South American river basins. Chapter 3.22. Freshwater Fisheries Ecology. First Edition. Edited by John F. Craig. Pp. 311-348.

CCI e INCODER. 2011. Base de datos de volúmenes de pesca desembarcados 2011. Corporación Colombia Internacional e Instituto Colombiano de Desarrollo Rural. Bogotá, D.C., Colombia.

Crossman, N. D., B. Burkhard, S. Nedkov, S. Willemen, L. Petz, et al. 2013. A blueprint for mapping and modelling ecosystem services. Ecosystem Services 4:4-14.

Daily, G. C., S. Polasky, J. Goldstein, P. M. Kareiva, H. Mooney, et al. 2009. Ecosystem services in decision making: time to deliver. Frontiers in Ecology and the Environment 7(1):21-28.

Egoh, B., E. G. Drakou, M. B. Dunbar, J. Maes, and L. Willemen. 2012. Indicators for mapping ecosystem services: a review. Report EUR 25456 EN. JRC scientific and policy reports. European Commission. Italia. Pp. 113.

ESRI. 2013. ArcGIS Desktop: Release 10.2. Redlands, CA: Environmental Systems Research Institute.

FAO. 2016. El estado mundial de la pesca y la acuicultura 2016. Departamento de pesca y acuicultura de la FAO. Organización de las Naciones Unidas para la Agricultura y la Alimentación. Roma, Italia. Pp. 226.

Fisher, B., R. K. Turner, and P. Morling. 2009. Defining and classifying ecosystem services for decision making. Ecological Economics 68(3):643-53.

Flórez-Ayala, C., L. Estupiñán-Suárez, S. Rojas, C. Aponte, M. Quiñones, et al. 2015. El entramado anfibio. Colombia y su naturaleza anfibia. Capítulo III Pp. 56-69 en: U. Jaramillo, J. Cortés-Duque y C. Flórez (eds.). Colombia anfibia. Un país de humedales. Volumen 1. Instituto de investigación de recursos biológicos Alexander von Humboldt, Bogotá D.C., Colombia. Pp. 139.

Galvis, G., and J. I. Mojica. 2007. The Magdalena River fresh water fishes and fisheries. Aquatic Ecosystem Health and Management Society 10(2):127-39.

García-Nieto, A. P., M. García-Llorente, I. Iniesta-Arandia, and B. Martín-López. 2013. Mapping forest ecosystem services: From providing units to beneficiaries. Ecosystem Services 4:126-138.

Gu, Z., L. Gu, R. Elis, M. Schlesner, and B. Brors. 2014. Circlize implements and enhances circular visualization in R. Bioinformatics 19:2811-2812.

Gutiérrez, F. P., C. Barreto, and B. Mancilla. 2011a. Diagnóstico de la pesquería en la cuenca Magdalena-Cauca. Capítulo 1. Pp. 35-73 en: C. A. Lasso, F. P. Gutiérrez, M. A. Morales-Betancourt, E. Agudelo, H. Ramírez-Gil y R. E. Ajiaco-Martínez (eds.). Pesquerías continentales de Colombia: cuencas del Magdalena-Cauca, Sinú, Canalete, Atrato, Orinoco, Amazonas y vertiente del Pacífico. Serie Editorial Recursos Hidrobiológicos y Pesqueros Continentales de Colombia. Instituto de Investigación de Recursos Biológicos Alexander von Humboldt, Bogotá, D.C.

Gutiérrez, F. P., C. A. Lasso, and M. A. Morales-Betancourt. 2011b. Introducción. Pp. 29-32en: C. A. Lasso, F. P. Gutiérrez, M. A. Morales-Betancourt, E. Agudelo, H. Ramírez-Gil y R. E. Ajiaco-Martínez (eds.). Pesquerías continentales de Colombia: cuencas del Magdalena-Cauca, Sinú, Canalete, Atrato, Orinoco, Amazonas y vertiente del Pacífico. Serie Editorial Recursos Hidrobiológicos y Pesqueros Continentales de Colombia. Instituto de Investigación de Recursos Biológicos Alexander von Humboldt, Bogotá, D.C.

Gutiérrez, F. P., J. Rodríguez, and C. A. Lasso. 2011c. Introducción. Pp. 19-21en: C. A. Lasso, E. Agudelo, L. JiménezSegura, H. Ramírez-Gil, M. Morales-Betancourt, et al. (eds.). Catálogo de los recursos pesqueros continentales de Colombia. Serie Editorial Recursos Hidrobiológicos y Pesqueros Continentales de Colombia. Instituto de Investigación de Recursos Biológicos Alexander von Humboldt, Bogotá, D.C. Pp. 715.

James, S. A., P. Petry, and R. E. Reis. 2011. Major biogeographic and phylogenetic patterns. Pp. 21-57 en: S. A. James and R. E. Reis (eds.). Historical Biogeography of Neotropical Freshwater Fishes. University of California Press. Los Angeles, USA.

Jaramillo, U. 2015. La naturaleza de los humedales. El pulso de inundación. Los latidos del agua. Pp. 30-31 en: U. Jaramillo, J. Cortés-Duque y C. Flórez (eds.). Colombia anfibia. Un país de humedales. Volumen 1. Bogotá D.C.

Lasso, C. A., E. Agudelo, L. Jiménez-Segura, H. Ramírez-Gil, M. Morales-Betancourt, et al. 2011. Catálogo de los recursos pesqueros continentales de Colombia. Serie Editorial Recursos Hidrobiológicos y Pesqueros Continentales de Colombia. Instituto de Investigación de Recursos Biológicos Alexander von Humboldt. Bogotá D.C., Colombia. Pp. 715.

Martínez-Harms, M. J., and P.Balvanera. 2012. Methods for mapping ecosystem service supply: a review. International Journal of Biodiversity Science, Ecosystem Services and Management 8(1-2):17-25.

Martín-López, B. 2012. Evaluación de los servicios de los ecosistemas suministrados por cuencas hidrográficas: una aproximación socio-ecológica. Pp. 1-14en: Observatorio del Agua Fundación Botín (eds.). $9^{\circ}$ Seminario Nacional: 
Agua y Naturaleza. Madrid, España.

Maynard, S., D. James, A. Davidson. 2010. The development of an ecosystem services framework for South East Queensland.Environmental Management 45(5):881-895.

MEA. 2005. Los Ecosistemas y el Bienestar Humano: Humedales y Agua. Informe de Síntesis. World Resources Institute. Washington, D. C. Pp. 68.

Mojica, J. I., J. S. Usma, R. Álvarez, and C. A. Lasso. 2012. Libro rojo de peces dulceacuícolas de Colombia 2012. Instituto de Investigación de Recursos Biológicos Alexander von Humboldt, Instituto de Ciencias Naturales de la Universidad Nacional de Colombia, WWF Colombia y Universidad de Manizales, Bogotá, D. C., Colombia. Pp. 319.

Mubareka, S., J. Maes, C. Lavalle, and A. de Roo. 2013. Estimation of water requirements by livestock in Europe. Ecosystem Services 4(0):139-145.

Núñez-Avellaneda, M., Z. Y. Marín, J. C. Alonso, E. Ríos, C. Andrade-Sossa, et al. 2006. Los ambientes de pesca en la frontera Colombo-Peruana del río Putumayo Pp. 29-42 en: E. Agudelo, J. C. Alonso, L. A. Moya (eds.). Perspectivas para el ordenamiento de la pesca y la acuicultura en el área de integración fronteriza colombo-peruana del río Putumayo. Instituto Amazónico de Investigaciones Científicas - SINCHI e Instituto Nacional de Desarrollo INADE. Bogotá D.C., Colombia. Pp. 85.

R Development Core Team. 2011. R: A language and environment for statistical computing. Vienna, Austria: the R Foundation for Statistical Computing. Available online at www.R-project.org.

Ramírez-Gil, H. y R. E. Ajiaco-Martínez. 2011. Diagnóstico de la pesquería en la cuenca del Orinoco Capítulo 6. Pp.169-198 en: C. A. Lasso, F. P. Gutiérrez, M. A. Morales-Betancourt, E. Agudelo, H. Ramírez-Gil, R. E. Ajiaco-Martínez (eds.). Pesquerías continentales de Colombia: cuencas del Magdalena-Cauca, Sinú, Canalete, Atrato, Orinoco, Amazonas y vertiente del Pacífico. Serie Editorial Recursos Hidrobiológicos y Pesqueros Continentales de Colombia. Instituto de Investigación de Recursos Biológicos Alexander von Humboldt, Bogotá, D.C.

Syrbe, R. U., and U. Walz. 2012. Spatial indicators for the assessment of ecosystem services: Providing, benefiting and connecting areas and landscape metrics. Ecological Indicators2 1:80-88.

Jaramillo, U., J. Cortés-Duque, C. Flórez. 2015. Colombia Anfibia. Un país de humedales. Volumen 1. Instituto de investigación de recursos biológicos Alexander von Humboldt. Bogotá D.C., Colombia. Pp. 140.

Valderrama, M. 2011. Prólogo. Pp. 13-15 en: C. A. Lasso, F. P. Gutiérrez, M. A. Morales-Betancourt, E. Agudelo, H. Ramírez-Gil, R. E. Ajiaco-Martínez (eds.). Pesquerías continentales de Colombia: cuencas del Magdalena-Cauca, Sinú, Canalete, Atrato, Orinoco, Amazonas y vertiente del Pacífico. Serie Editorial Recursos Hidrobiológicos y Pesqueros Continentales de Colombia. Instituto de Investigación de Recursos Biológicos Alexander von Humboldt, Bogotá, D.C. 